Supplement of The Cryosphere, 12, 453-476, 2018 https://doi.org/10.5194/tc-12-453-2018-supplement (C) Author(s) 2018. This work is distributed under the Creative Commons Attribution 4.0 License.

(c) (1)

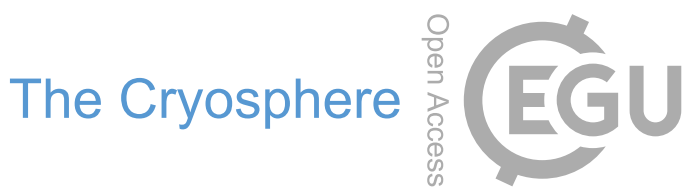

Supplement of

\title{
Tidal influences on a future evolution of the Filchner-Ronne Ice Shelf cavity in the Weddell Sea, Antarctica
}

Rachael D. Mueller et al.

Correspondence to: Rachael D. Mueller (mueller@esr.org)

The copyright of individual parts of the supplement might differ from the CC BY 4.0 License. 
Supplement:

ROMS Model Paramter selection Physical Parameters, Grid: 01

$\begin{array}{rll}======================== \\ 3153600 & \text { ntimes } & \text { Number of timesteps for 3-D equations. } \\ 200 & \text { dt } & \text { Timestep size (s) for 3-D equations. } \\ 30 & \text { ndtfast } & \text { Number of timesteps for 2-D equations between each 3D timestep. } \\ 1 & \text { ERstr } & \text { Starting ensemble/perturbation run number. } \\ 1 & \text { ERend } & \text { Ending ensemble/perturbation run number. } \\ 0 & \text { nrrec } & \text { Number of restart records to read from disk. } \\ \text { T } & \text { LcycleRST } & \text { Switch to recycle time-records in restart file. } \\ 12960 & \text { nRST } & \text { Number of timesteps between the writing of data into restart fields. } \\ 40 & \text { ninfo } & \text { Number of timesteps between print of information to standard output. } \\ \text { T } & \text { ldefout } & \text { Switch to create a new output NetCDF file(s). } \\ 157680 & \text { nHIS } & \text { Number of timesteps between the writing fields into history file. } \\ 1 & \text { ntsAVG } & \text { Starting timestep for the accumulation of output time-averaged data. } \\ 12960 & \text { nAVG } & \text { Number of timesteps between the writing of time-averaged data into averages file. } \\ 157680 & \text { ndefAVG } & \text { Number of timesteps between creation of new time-averaged file. } \\ 2000000 & \text { ntsDIA } & \text { Starting timestep for the accumulation of output time-averaged diagnostics data. } \\ 2000000 & \text { nDIA } & \text { Number of timesteps between the writing of time-averaged data into diagnostics file. } \\ 1.00 \mathrm{E}+00 & \text { nl_tnu2(01) } & \text { NLM Horizontal, harmonic mixing coefficient (m2/s) for tracer 01: temp } \\ 1.00 \mathrm{E}+00 & \text { nl_tnu2(02) } & \text { NLM Horizontal, harmonic mixing coefficient (m2/s) for tracer 02: salt } \\ 5.00 \mathrm{E}+00 & \text { nl_visc2 } & \text { NLM Horizontal, harmonic mixing coefficient (m2/s) for momentum. } \\ 1.00 \mathrm{E}-05 & \text { Akt_bak(01) } & \text { Background vertical mixing coefficient (m2/s) for tracer 01: temp } \\ 1.00 \mathrm{E}-05 & \text { Akt_bak(02) } & \text { Background vertical mixing coefficient (m2/s) for tracer 02: salt } \\ 5.00 \mathrm{E}-05 & \text { Akv_bak } & \text { Background vertical mixing coefficient (m2/s) for momentum. } \\ 3.00 \mathrm{E}-04 & \text { rdrg } & \text { Linear bottom drag coefficient (m/s). } \\ 2.50 \mathrm{E}-03 & \text { rdrg2 } & \text { Quadratic bottom drag coefficient. } \\ & & \end{array}$




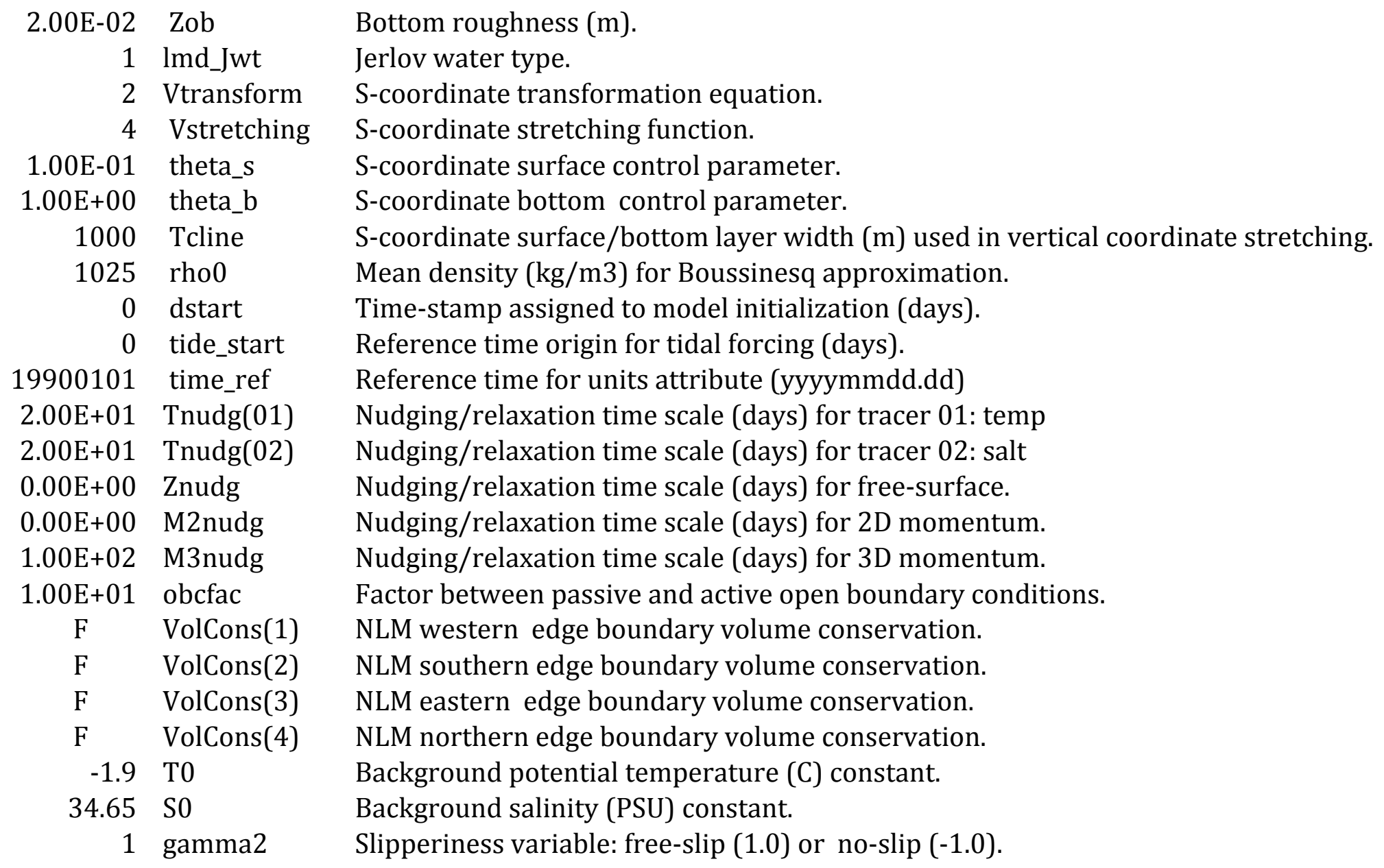

Tile partition information for Grid 01: 0205x0211x0024 tiling: 008x004

Lateral Boundary Conditions: NLM

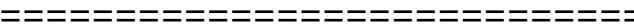


Variable

zeta
ubar
vbar
u
v
temp
salt

Activated C-preprocessing Options:

=ニニニニニニニニニニニニニニニニニニニニニニニニニニニ=

\section{WS}

ADD_FSOBC

ADD_M2OBC

ANA_BSFLUX

ANA_BTFLUX

ANA_FSOBC

ANA_INITIAL

ANA_M2OBC

ANA_M30BC

ANA_SMFLUX

ANA_SRFLUX

ANA_SSFLUX

ANA_STFLUX

ASSUMED_SHAPE

AVERAGES

CURVGRID

\section{ROMS/TOMS 3.6 - WS sub}

Adding tidal elevation to processed OBC data. Adding tidal currents to processed OBC data.

Analytical kinematic bottom salinity flux.

Analytical kinematic bottom temperature flux. Analytical free-surface boundary conditions. Analytical initial conditions.

Analytical 2D momentum boundary conditions. Analytical 3D momentum boundary conditions. Analytical kinematic surface momentum flux. Analytical kinematic shortwave radiation flux. Analytical kinematic surface salinity flux. Analytical kinematic surface temperature flux.

Using assumed-shape arrays.

Writing out time-averaged nonlinear model fields.

Orthogonal curvilinear grid. 


$\begin{array}{ll}\text { DIAGNOSTICS_TS } & \text { Computing and writing tracer diagnostic terms. } \\ \text { DJ_GRADPS } & \text { Parabolic Splines density Jacobian (Shchepetkin, 2002). } \\ \text { DOUBLE_PRECISION } & \begin{array}{c}\text { Double precision arithmetic. } \\ \text { Include Ice Shelf Cavities. }\end{array} \\ \text { ICESHELF } & \text { Include 3eq Ice Shelf Thermodynamics. } \\ \text { ICESHELF_3EQ } & \text { LMD convective mixing due to shear instability. } \\ \text { LMD_CONVEC } & \text { Large/McWilliams/Doney interior mixing. } \\ \text { LMD_MIXING } & \text { LMD convective nonlocal transport. } \\ \text { LMD_NONLOCAL } & \text { LMD diffusivity due to shear instability. } \\ \text { LMD_RIMIX } & \text { KPP surface boundary layer mixing. } \\ \text { LMD_SKPP } & \text { Land/Sea masking. } \\ \text { MASKING } & \text { Mixing of tracers along geopotential surfaces. } \\ \text { MIX_GEO_TS } & \text { Mixing of momentum along constant S-surfaces. } \\ \text { MIX_S_UV } & \text { MPI distributed-memory configuration. } \\ \text { MPI } & \text { Nonlinear Model. } \\ \text { NONLINEAR } & \text { Nonlinear Equation of State for seawater. } \\ \text { NONLIN_EOS } & \text { Processing perfect restart variables. } \\ \text { PERFECT_RESTART } & \text { Power-law shape time-averaging barotropic filter. } \\ \text { POWER_LAW } & \text { Time profiling activated . } \\ \text { PROFILE } & \text { Ramping tidal forcing for one day. } \\ \text { RAMP_TIDES } & \text { Double precision fields in restart NetCDF file. } \\ \text { !RST_SINGLE } & \text { Using salinity. } \\ \text { SALINITY } & \text { Solving 3D Primitive Equations. } \\ \text { SOLVE3D } & \text { Conservative parabolic spline reconstruction. } \\ \text { SPLINES } & \text { Add tidal elevation to SSH climatology. } \\ \text { SSH_TIDES } & \text { Third-order upstream horizontal advection of tracers. } \\ \text { TS_U3HADVECTION } & \text { Fourth-order centered vertical advection of tracers. } \\ \text { TS_C4VADVECTION } & \text { Harmonic mixing of tracers. } \\ \text { TS_DIF2 } & \text { Advection of momentum. } \\ \text { UV_ADV } & \text { Coriolis term. } \\ \text { UV_COR } & \end{array}$


UV_U3HADVECTION

UV_C4VADVECTION

UV_QDRAG

UV_TIDES

UV VIS2

VAR_RHO_2D
Third-order upstream horizontal advection of 3D momentum.

Fourth-order centered vertical advection of momentum.

Quadratic bottom stress.

Add tidal currents to 2D momentum climatologies.

Harmonic mixing of momentum.

Variable density barotropic mode. 